\title{
Atmosfera modificada e refrigeração para conservação pós-colheita de uva 'Niagara Rosada'
}

\author{
Patrícia Cia(1), Eliane Aparecida Benato(2), Silvia Regina de Toledo Valentini(2), Juliana Sanches ${ }^{(1)}$, \\ Francine Scolfaro Ponzo ${ }^{(3)}$, Daniela Flôres ${ }^{(4)}$ e Maurilo Monteiro Terra ${ }^{(5)}$
}

\begin{abstract}
(1)Instituto Agronômico (IAC), Centro de Engenharia e Automação, Rodovia Dom Gabriel P.B. Couto, Km 65, Caixa Postal 26, CEP 13201-970 Jundiaí, SP. E-mail: pcia@iac.sp.gov.br, jsanches@iac.sp.gov.br (2)Instituto de Tecnologia de Alimentos, Grupo de Engenharia e Pós-Colheita, Avenida Brasil, no 2.880, Caixa Postal 139, CEP 13070-178 Campinas, SP. E-mail: benato@ital.sp.gov.br, valentini@ital.sp.gov.br (3)IAC, Pós-Graduação em Agricultura Tropical e Subtropical, Avenida Barão de Itapura, no 1.481, Caixa Postal 28, CEP $13012-970$ Campinas, SP E-mail: fran_cca@yahoo.com.br (4)Universidade de São Paulo, Escola Superior de Agricultura Luiz de Queiroz, Avenida Pádua Dias, no 11, Caixa Postal 9, CEP 13418-900 Piracicaba, SP. E-mail: daniflor@esalq.usp.br ${ }^{(5)}$ IAC, Centro de Frutas, Avenida Luiz Pereira dos Santos, №1.500, CEP 13214-800 Jundiaí, SP. E-mail: mmterra@iac.sp.gov.br.
\end{abstract}

Resumo - O objetivo deste trabalho foi avaliar os efeitos da atmosfera modificada na conservação pós-colheita da uva 'Niagara Rosada' armazenada sob refrigeração, em dois experimentos. No primeiro experimento avaliou-se o acondicionamento de cachos nas seguintes embalagens: papelão ondulado (testemunha); tereftalato de polietileno (PET); cloreto de polivinila (PVC) $17 \mu \mathrm{m}$; polietileno linear de baixa densidade (PELBD) $25 \mu \mathrm{m}$; e PELBD $50 \mu \mathrm{m}$. Em outro experimento, avaliaram-se os sistemas de acondicionamento: sacolas de plástico abertas (testemunha); polietileno de baixa densidade (PEBD) $25 \mu \mathrm{m}$; PEBD $25 \mu \mathrm{m}$, com injeção de mistura gasosa $\left(21 \% \mathrm{O}_{2} / 5 \% \mathrm{CO}_{2}\right)$; PEBD $25 \mu \mathrm{m}$ $\left(21 \% \mathrm{O}_{2} / 10 \% \mathrm{CO}_{2}\right)$; PEBD $25 \mu \mathrm{m}\left(21 \% \mathrm{O}_{2} / 20 \% \mathrm{CO}_{2}\right)$. Os cachos foram armazenados a $1 \pm 1^{\circ} \mathrm{C}$ e $90 \pm 5 \%$ de umidade relativa (UR) por 28 dias, seguido de armazenamento em condições do ambiente $\left(25 \pm 2^{\circ} \mathrm{C}\right.$ e $80 \pm 5 \%$ UR). Os cachos foram avaliados quanto à perda de massa de matéria fresca, firmeza, cor das bagas, esbagoamento, sólidos solúveis totais (SST), acidez titulável (AT), relação SST/AT e incidência de podridões. O filme PELBD $50 \mu \mathrm{m}$, a partir do $14^{\circ}$ dia a $1{ }^{\circ} \mathrm{C}$, seguido por mais três dias a $25^{\circ} \mathrm{C}$, causou a fermentação dos cachos. As embalagens PELBD $25 \mu \mathrm{m}$, com ou sem injeção de mistura gasosa, e PVC $17 \mu \mathrm{m}$ reduzem a perda de massa de matéria fresca dos cachos, mas não reduzem o esbagoamento e a incidência de podridões.

Termos para indexação: Vitis labrusca, esbagoamento, podridões.

\section{Modified atmosphere and cold storage for postharvest conservation of 'Niagara Rosada' table grape}

\begin{abstract}
The objective of this work was to evaluate the effect of the modified atmosphere, on the storage life of 'Niagara Rosada' Table grape kept under refrigeration in two experiments. In the first one, grape clusters stored in different package materials were evaluated: corrugated cardboard boxes, polyethylene tereftalate (PET); 17- $\mu \mathrm{m}$ polyvinyl chloride (PVC); 25- $\mu \mathrm{m}$ low density linear polyethylene film (PELDB); and 50- $\mu \mathrm{m}$ PELDB. On a second assay, different packaging systems were evaluated: opened plastic bags; $25-\mu \mathrm{m}$ low density polyethylene film (PEBD); 25- $\mu \mathrm{m}$ PEBD, with injection of gas mixture $\left(21 \% \mathrm{O}_{2} / 5 \% \mathrm{CO}_{2}\right), 25-\mu \mathrm{m}$ PEBD $\left(21 \% \mathrm{O}_{2} / 10 \% \mathrm{CO}_{2}\right)$; $25-\mu \mathrm{m}$ PEBD $\left(21 \% \mathrm{O}_{2} / 20 \% \mathrm{CO}_{2}\right)$. The grape clusters were stored at $1 \pm 1{ }^{\circ} \mathrm{C}$ and $90 \pm 5 \%$ relative humidity (RH) for 28 days, followed by a storage period at room conditions at $25 \pm 2^{\circ} \mathrm{C}$ and $80 \pm 5 \% \mathrm{RH}$. Clusters were evaluated for weight loss, firmness, color of the berries, percentage of detached berries, total soluble solids (SST), titratable acidity (TA), SST/TA, and rot incidence. Polyethylene film of $50-\mu \mathrm{m}$, from the $14^{\text {th }}$ day at $1{ }^{\circ} \mathrm{C}$, with an additional period of 3 days at $25^{\circ} \mathrm{C}$, caused fruit fermentation. The $25-\mu \mathrm{m}$ PELBD, with or without gas injection, and $17-\mu \mathrm{m}$ PVC films reduce clusters weight loss, but do not reduce berry detaching and rot incidence .
\end{abstract}

Index terms: Vitis labrusca, detached berries, rots.

\section{Introdução}

O Estado de São Paulo destaca-se como o maior produtor nacional de uva de mesa, com produção anual de 189,7 mil toneladas. As cultivares de uva comum, representadas principalmente pela 'Niagara Rosada', correspondem a 49,1\% da produção no Estado (Instituto de Economia Agrícola, 2007). Por ter custo de produção menor que o da uva fina de mesa, características de rusticidade que exigem um número menor de pulverizações de defensivos agrícolas e excelente aceitação pelo mercado consumidor, o cultivo da videira 'Niagara' está se expandindo por todos os importantes pólos vitícolas do Brasil (Tecchio et al., 2009). No entanto, a produção concentra-se nos meses de dezembro a fevereiro, o que ocasiona

Pesq. agropec. bras., Brasília, v.45, n.10, p.1058-1065, out. 2010 
picos de preços em setembro-outubro e março-abril (Pommer et al., 2003).

Nos últimos anos, os viticultores verificaram uma queda na rentabilidade da cultura, o que acarretou a necessidade de se adequarem as técnicas de cultivo, para redução dos custos de produção e das perdas pós-colheita (Tecchio et al., 2009). Segundo Maia (2002), o período entre a colheita e a venda da 'Niagara Rosada', no varejo, deve ser o menor possível, em razão dos problemas de esbagoamento e incidência de podridões.

Na pós-colheita, importantes medidas de controle de podridões incluem manuseio cuidadoso, resfriamento rápido e uso de dióxido de enxofre $\left(\mathrm{SO}_{2}\right)$, aliadas ao armazenamento refrigerado, sob temperaturas próximas a $0^{\circ} \mathrm{C}$ (Camili et al., 2007). Além de eficiente na redução da incidência de podridões, o tratamento com $\mathrm{SO}_{2}$ permite que os cachos sejam mantidos em condições de alta umidade relativa. Entretanto, o $\mathrm{SO}_{2}$ pode causar danos às bagas, como o branqueamento, e os resíduos deixados pelo produto não são aceitos por alguns mercados consumidores (Chervin et al., 2005).

Atualmente, tem sido dada ênfase para o desenvolvimento de técnicas alternativas de controle de podridões, como o uso de tratamentos físicos, para a manutenção da qualidade e a proteção de frutos em pós-colheita, que garantam a segurança do produto e não coloquem em risco a saúde do consumidor. Entre os métodos físicos que podem ser empregados, para o aumento do período de conservação dos frutos e controle de podridões pós-colheita de uva, destacamse a refrigeração e a atmosfera modificada (AM) (Cia \& Benato, 2006).

A redução da taxa respiratória e o atraso dos processos bioquímicos da maturação e senescência podem ser obtidos pela técnica de AM (Kader et al., 1989). Esta técnica tem por objetivo estender o período de conservação das frutas, pela redução do desenvolvimento de podridões, com atuação direta e indireta sobre os patógenos (Cia \& Benato, 2006). Para que a técnica de AM seja eficiente, o processo de senescência dos frutos deve ser atrasado, sem reduzir a qualidade. Com o atraso da senescência, o fruto pode manter sua habilidade para produzir compostos antifúngicos, normalmente presentes em frutos jovens, o que evita o desenvolvimento de podridões. Estes efeitos acarretam inibição da doença e atraso na transformação de infecções quiescentes em infecções ativas.

No Brasil, poucos foram os estudos realizados que abordaram a utilização de atmosfera modificada ou controlada para o armazenamento de uvas de mesa. Sob tal aspecto, Brackmann et al. (2000) relataram que a utilização de altas concentrações de $\mathrm{CO}_{2}$, durante o armazenamento refrigerado de uvas 'Dona Zilá' e 'Tardia de Caxias', mostrou-se eficiente na redução de podridões pós-colheita. Chitarra \& Chitarra (2005) recomendam concentrações de 2 a $5 \%$ de $\mathrm{O}_{2}+1$ a $3 \%$ de $\mathrm{CO}_{2}$ para o armazenamento de uvas sob refrigeração. Crisosto et al. (2002) avaliaram os efeitos de baixas concentrações de $\mathrm{O}_{2}$ e altas concentrações de $\mathrm{CO}_{2}$ como substituto ao $\mathrm{SO}_{2}$, para o controle de Botrytis cinerea em uvas 'Redglobe', e concluíram que a utilização de $10 \%$ de $\mathrm{CO}_{2}$, independentemente da concentração de $\mathrm{O}_{2}(3,6$ ou $12 \%)$, reduziu a incidência de $B$. cinerea, durante o armazenamento dos cachos a $1^{\circ} \mathrm{C}$ por oito semanas, seguido por mais três dias a $20^{\circ} \mathrm{C}$; porém, concentrações acima de $10 \%$ de $\mathrm{CO}_{2}$ aceleraram o escurecimento da ráquis, o que prejudicou a aparência dos cachos. Castro et al. (1999) analisaram a taxa de esbagoamento, a perda de massa dos cachos, o bom aspecto do cacho e do engaço, e constataram que entre as embalagens avaliadas (polietileno de 0,05 e 0,08 mm de espessura, e sacolas de plástico sanfonadas), a embalagem sacola de plástico foi a adequada para conservar uvas 'Itália' da região de Jales, por até seis semanas a $4^{\circ} \mathrm{C}$.

Assim, o desenvolvimento de tecnologias pós-colheita, adequadas para melhor conservação dos frutos e que, ao mesmo tempo, sejam eficientes na redução das perdas decorrentes de problemas fitossanitários e reduzam o uso de defensivos agrícolas, são de grande importância.

O objetivo deste trabalho foi avaliar os efeitos da atmosfera modificada ativa e passiva, na conservação pós-colheita de uvas 'Niagara Rosada' mantidas sob refrigeração.

\section{Material e Métodos}

Dois experimentos foram realizados para a avaliação da atmosfera modificada na conservação pós-colheita de uva 'Niagara Rosada'. Cachos de uva 'Niagara Rosada', provenientes de Louveira (Experimento I) e Jundiaí (Experimento II), Estado de São Paulo, foram colhidos

Pesq. agropec. bras., Brasília, v.45, n.10, p.1058-1065, out. 2010 
com teor de sólidos solúveis totais de 12 e 13,5\%, para os Experimentos I e II, respectivamente, e transportados ao laboratório de pós-colheita, onde foram selecionados quanto à ausência de defeitos, podridões e uniformidade de cor. No primeiro experimento, os cachos com aproximadamente 750 geemnúmero de três porrepetição, foram acondicionados nas seguintes embalagens: papelão ondulado (testemunha), politereftalato de etileno (PET), com tampa e fundo perfurados; cloreto de polivinila (PVC) $17 \mu \mathrm{m}$; polietileno linear de baixa densidade (PELBD) $25 \mu \mathrm{m}$; e PELBD $50 \mu \mathrm{m}$. O filme de PVC $17 \mu \mathrm{m}$, o PELBD $25 \mu \mathrm{m}$ e o de $50 \mu \mathrm{m}$ foram usados paraenvolverbandejas depoliestireno. Frutos testemunha não foram acondicionados em filmes de plástico. Os cachos testemunha, bem como aqueles envoltos em diferentes filmes de plástico, foram acondicionados em caixas de papelão ondulado. No segundo experimento, os cachos com aproximadamente $550 \mathrm{~g}$ e em número de dois por repetição, foram acondicionados em diferentes sistemas de embalagem, que se constituíram de: sacolas de plástico abertas (testemunha); polietileno de baixa densidade (PEBD) $25 \mu \mathrm{m}$; PEBD $25 \mu \mathrm{m}$, com injeção de mistura gasosa $\left(21 \% \mathrm{O}_{2} / 5 \% \mathrm{CO}_{2}\right)$; PEBD $25 \mu \mathrm{m}$ $\left(21 \% \mathrm{O}_{2} / 10 \% \mathrm{CO}_{2}\right)$; PEBD $25 \mu \mathrm{m}\left(21 \% \mathrm{O}_{2} / 20 \% \mathrm{CO}_{2}\right)$. Os frutos testemunha, bem como aqueles envoltos em diferentes filmes de plástico, foram acondicionados em caixas de papelão ondulado. Para a injeção de mistura gasosa nas embalagens de polietileno, utilizouse termosseladora Selovac 200B (Selovac, São Paulo, Brasil), por meio de cilindros de alta pressão, com misturas conhecidas. No primeiro experimento, os cachos foram armazenados em câmaras de refrigeração $\left(1 \pm 1^{\circ} \mathrm{C}\right.$ e $90 \pm 5 \%$ UR), por $7,14,21$ e 28 dias, e após cada período, foram transferidos para condições do ambiente $\left(25 \pm 2^{\circ} \mathrm{C}\right.$ e $80 \pm 5 \%$ UR). As análises foram realizadas semanalmente, após os cachos terem sido transferidos para condições do ambiente por três dias. No segundo experimento, os cachos foram avaliados após 15 dias de armazenamento refrigerado, seguido por mais cinco dias a $25^{\circ} \mathrm{C}$. Neste ensaio, no momento da transferência dos cachos para as condições do ambiente, metade dos cachos foi mantida nas embalagens e a outra parte foi retirada das condições de atmosfera modificada e transferida para sacolas de plástico.

Foram avaliados o atributos de qualidade conforme descrição a seguir. Incidência de podridões (\%), determinada pela diferença de massa obtida na pesagem dos cachos e das bagas que apresentavam sintomas de podridão, por meio do cálculo $\mathrm{P}(\%)=$ (massa de bagas podres/massa do cacho de uva) x 100 (Cia et al., 2009). Esbagoamento (\%), calculado pela diferença de massa obtida pela pesagem dos cachos e das bagas caídas após leve agitação manual dos cachos, por cinco vezes. Firmeza das bagas $(\mathrm{N})$, determinada em texturômetro TA-XT2 (Stable Micro Systems, Surrey, UK), com probe cilíndrico de alumínio (35 $\mathrm{mm}$ ), com velocidade de $2 \mathrm{~mm} \mathrm{~s}^{-1}$ e profundidade de compressão de $2 \mathrm{~mm}$, em duas bagas da porção basal, duas da região mediana e duas da região apical, por cacho. Coloração da casca, obtida em colorímetro Minolta CR 300 (Konica Minolta, Osaka, Japão), com sistema CIELab dos parâmetros de luminosidade $\left(\mathrm{L}^{*}\right)$, cor verde $\left(-\mathrm{a}^{*}\right)$, cor vermelha $\left(+\mathrm{a}^{*}\right)$, tendo-se efetuado a leitura em seis bagas por cacho. Calculou-se a diferença de coloração total entre padrão e amostras, por meio da fórmula:

$$
\Delta \mathrm{E}=\left[(\Delta \mathrm{L})^{2}+(\Delta \mathrm{a})^{2}+(\Delta \mathrm{b})^{2}\right]^{1 / 2} .
$$

O teor de sólidos solúveis totais (\%) foi obtido em refratômetro digital Schmidt-Haensch (Schmidt + Haensch $\mathrm{GmbH} \&$ Co., Berlin, Alemanha). A acidez titulável, expressa em \% de ácido tartárico, foi determinada por titulação com $\mathrm{NaOH}(0,1 \mathrm{~N})$ até $\mathrm{pH}$ 8,1, em pHmetro Mettler Toledo 320 (Carvalho et al., 1990).

Além dessas análises avaliou-se semanalmente, a composição gasosa $\left(\mathrm{O}_{2} / \mathrm{CO}_{2}\right)$ no espaço-livre das embalagens, com auxílio de um analisador de gases Mocon (Mocon, Minneapolis, EUA), com alíquotas de $30 \mathrm{~mL}$ de gás, obtidas do interior das embalagens com a coleta via septo.

Utilizou-se o delineamento experimental inteiramente casualizado, com quatro (Experimento I) ou cinco repetições (Experimento II), compostas por três (Experimento I) ou dois cachos (Experimento II) como unidade experimental. As médias dos dados obtidos foram submetidas à análise de variância e comparados pelo teste de Tukey a 5\% de probabilidade, pelo programa Sisvar (Ferreira, 2005).

\section{Resultados e Discussão}

No primeiro experimento, durante o armazenamento refrigerado, as concentrações médias de $\mathrm{O}_{2}$ após o estabelecimento do equilíbrio da atmosfera, foram 20,53, 16,27 e 8,40\% com uso de PVC $17 \mu \mathrm{m}$, 

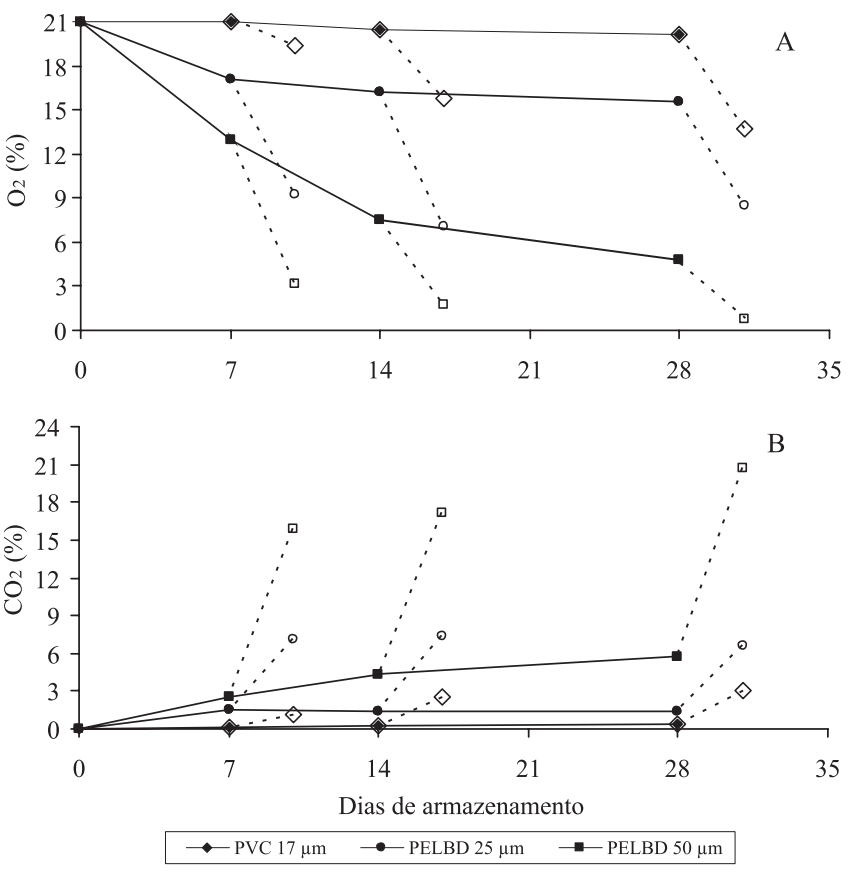

Figura 1. Concentrações de $\mathrm{O}_{2}(\mathrm{~A})$ e $\mathrm{CO}_{2}$ (B) no espaço-livre das embalagens - PVC $17 \mu \mathrm{m}$, cloreto de polivinila; PELBD 25 $\mu \mathrm{m}$ e PELBD $50 \mu \mathrm{m}-$, durante o armazenamento refrigerado de uvas 'Niagara Rosada' $\left(1^{\circ} \mathrm{C}\right.$ e $90 \%$ de umidade relativa), seguido de um período de três dias sob condições do ambiente $\left(25^{\circ} \mathrm{C}\right.$ e $80 \%$ de umidade relativa, linha pontilhada). Experimento I.
PELBD $25 \mu \mathrm{m}$ e PELBD $50 \mu \mathrm{m}$, respectivamente, enquanto as concentrações de $\mathrm{CO}_{2}$ foram $0,27,1,50$ e 4,23\% (Figura 1). Após a transferência dos frutos para armazenamento a $25^{\circ} \mathrm{C}$, observaram-se reduções nas concentrações de $\mathrm{O}_{2}$ de $16,30,8,27$ e 1,87\% com PVC $17 \mu \mathrm{m}$, PELBD $25 \mu \mathrm{m}$ e PELBD $50 \mu \mathrm{m}$, respectivamente, e aumento imediato nas de $\mathrm{CO}_{2}$ de 2,20, 7,00 e 17,97\% com PVC $17 \mu \mathrm{m}$, PELBD $25 \mu \mathrm{m}$, PELBD $50 \mu \mathrm{m}$, respectivamente. Portanto, o filme PVC $17 \mu \mathrm{m}$ alterou ligeiramente a composição gasosa, principalmente durante o armazenamento refrigerado dos cachos, enquanto o filme PELBD $50 \mu \mathrm{m}$ reduziu acentuadamente as concentrações de $\mathrm{O}_{2}$ e elevou as de $\mathrm{CO}_{2}$ bruscamente, principalmente após transferência para $25^{\circ} \mathrm{C}$. No entanto, o filme PELBD $25 \mu \mathrm{m}$ alterou significativamente as concentrações de $\mathrm{O}_{2}$ e $\mathrm{CO}_{2}$, durante o armazenamento refrigerado, e não promoveu riscos de anaerobiose após a transferência dos cachos para $25^{\circ} \mathrm{C}$. Sob tal aspecto, Crisosto et al. (2002) relataram que concentrações de $\mathrm{CO}_{2}$ acima de $10 \%$ causam escurecimento de ráquis em uvas 'Redglobe'. Deng et al. (2006) constataram que o armazenamento de uvas 'Kyoho', sob condições de atmosfera controlada $\left(4 \% \mathrm{O}_{2}+9 \% \mathrm{CO}_{2}\right)$, manteve a qualidade dos frutos por 60 dias.

Tabela 1. Esbagoamento, incidência de podridões, perda de massa de matéria fresca, teores de sólidos solúveis totais (SST) e acidez titulável (AT) de uvas 'Niagara Rosada', acondicionadas em atmosfera modificada passiva e armazenadas a $1{ }^{\circ} \mathrm{C}$ e $90 \%$ de umidade relativa (UR) por até 28 dias, seguidos de mais três dias sob condições do ambiente $\left(25^{\circ} \mathrm{C} \text { e } 80 \% \text { UR }\right)^{(1)}$.

\begin{tabular}{|c|c|c|c|c|c|c|c|}
\hline Tratamento & $\begin{array}{c}\text { Tempo de } \\
\text { armazenamento (dias) }\end{array}$ & $\begin{array}{c}\text { Esbagoamento }{ }^{(2)} \\
(\%)\end{array}$ & $\begin{array}{l}\text { Podridões }^{(2)} \\
(\%)\end{array}$ & $\begin{array}{l}\text { Perda de massa de } \\
\text { matéria fresca }(\%)\end{array}$ & $\begin{array}{l}\text { SST } \\
(\%)\end{array}$ & $\begin{array}{c}\mathrm{AT} \\
\text { (\% de ácido tartárico) }\end{array}$ & SST/AT \\
\hline- & 0 & 0 & 0 & 0 & 12,0 & 0,59 & 20,64 \\
\hline Testemunha & & $4,71 \mathrm{a}$ & $9,68 \mathrm{a}$ & $1,73 \mathrm{a}$ & $11,7 \mathrm{a}$ & $0,58 \mathrm{a}$ & $20,18 \mathrm{a}$ \\
\hline PET & & $1,46 \mathrm{a}$ & $7,92 \mathrm{a}$ & $1,24 \mathrm{~b}$ & $12,1 \mathrm{a}$ & $0,56 \mathrm{a}$ & $21,50 \mathrm{a}$ \\
\hline $\mathrm{PVC} 17 \mu \mathrm{m}$ & $7+3$ & $1,61 \mathrm{a}$ & $6,02 \mathrm{a}$ & $0,87 \mathrm{~b}$ & $11,5 \mathrm{a}$ & $0,64 \mathrm{a}$ & $18,09 \mathrm{a}$ \\
\hline PELBD $25 \mu \mathrm{m}$ & & $1,47 \mathrm{a}$ & $6,94 a$ & $0,39 \mathrm{c}$ & $11,6 \mathrm{a}$ & $0,55 \mathrm{a}$ & $21,08 \mathrm{a}$ \\
\hline PELBD $50 \mu \mathrm{m}$ & & $3,70 \mathrm{a}$ & $9,70 \mathrm{a}$ & $0,37 \mathrm{c}$ & $11,7 \mathrm{a}$ & $0,72 \mathrm{a}$ & $16,92 \mathrm{a}$ \\
\hline $\mathrm{CV}(\%)$ & & 36,01 & 28,32 & 19,85 & 4,28 & 14,05 & 12,28 \\
\hline Testemunha & & $5,89 \mathrm{a}$ & $10,28 \mathrm{a}$ & $3,10 \mathrm{a}$ & $12,2 \mathrm{a}$ & $0,46 \mathrm{a}$ & $26,74 a$ \\
\hline PET & & $1,94 b$ & $7,46 \mathrm{a}$ & $2,98 \mathrm{a}$ & $12,8 \mathrm{a}$ & $0,48 \mathrm{a}$ & $29,52 \mathrm{a}$ \\
\hline $\mathrm{PVC} 17 \mu \mathrm{m}$ & $14+3$ & $2,64 a b$ & $8,62 \mathrm{a}$ & $1,25 \mathrm{~b}$ & $12,1 \mathrm{a}$ & $0,41 \mathrm{a}$ & $33,77 \mathrm{a}$ \\
\hline PELBD $25 \mu \mathrm{m}$ & & $1,97 \mathrm{~b}$ & $9,68 \mathrm{a}$ & $0,46 \mathrm{c}$ & $12,1 \mathrm{a}$ & $0,30 \mathrm{a}$ & $41,03 \mathrm{a}$ \\
\hline PELBD $50 \mu \mathrm{m}$ & & $3,71 \mathrm{ab}$ & $11,44 a$ & $0,43 \mathrm{c}$ & $11,6 \mathrm{a}$ & $0,51 \mathrm{a}$ & $25,08 \mathrm{a}$ \\
\hline CV (\%) & & 22,27 & 24,74 & 13,15 & 5,10 & 29,83 & 34,02 \\
\hline Testemunha & & $4,93 a$ & $5,63 a$ & $2,85 a$ & $12,4 \mathrm{a}$ & $0,43 a$ & $28,96 a$ \\
\hline PET & & $3,45 \mathrm{a}$ & $11,70 \mathrm{a}$ & $3,34 \mathrm{a}$ & $11,9 \mathrm{abc}$ & $0,45 \mathrm{a}$ & $26,52 \mathrm{a}$ \\
\hline PVC $17 \mu \mathrm{m}$ & $28+3$ & $2,54 \mathrm{a}$ & $9,93 \mathrm{a}$ & $1,32 b$ & $12,1 \mathrm{ab}$ & $0,42 \mathrm{a}$ & $29,30 \mathrm{a}$ \\
\hline PELBD $25 \mu \mathrm{m}$ & & $3,20 \mathrm{a}$ & $7,72 \mathrm{a}$ & $0,47 \mathrm{c}$ & $11,6 \mathrm{bc}$ & $0,50 \mathrm{a}$ & $23,69 \mathrm{a}$ \\
\hline PELBD $50 \mu \mathrm{m}$ & & $6,27 \mathrm{a}$ & $14,23 \mathrm{a}$ & $0,56 \mathrm{c}$ & $11,3 \mathrm{c}$ & $0,50 \mathrm{a}$ & $23,03 a$ \\
\hline $\mathrm{CV}(\%)$ & & 43,53 & 22,97 & 16,39 & 2,85 & 13,14 & 12,45 \\
\hline
\end{tabular}


Quanto aos esbagoamento dos cachos e incidência de podridões (Tabela 1), constatou-se que as embalagens PET e PELBD $25 \mu \mathrm{m}$ reduziram significativamente (cerca de 67\%) o esbagoamento somente no $14^{\circ}$ dia de armazenamento sob refrigeração, seguido por mais três dias a $25^{\circ} \mathrm{C}$, enão influenciarama incidência de podridões. Provavelmente, a elevada incidência de podridões, nos cachos acondicionados sob condições de atmosfera modificada, pode ser resultado da elevada umidade relativa no interior das embalagens. A combinação entre a quantidade de cachos por embalagem, a espessura e os tipos de filmes empregados, bem como a temperatura de armazenamento $\left(1^{\circ} \mathrm{C}\right)$ não alteraram suficientemente a composição gasosa no interior das embalagens, ou seja, provavelmente não permitiram o acúmulo de altas concentrações de $\mathrm{CO}_{2}$ e redução dos níveis de $\mathrm{O}_{2}$ que pudessem atuar na redução da incidência de podridões nos frutos (Cia \& Benato, 2006).

Constatou-se que as embalagens avaliadas foram eficientes em limitar a perda de massa de matéria fresca dos cachos de uva (Tabela 1), com destaque para a PELBD. A redução da perda de massa de matéria fresca está diretamente relacionada à taxa de transmissão de vapor d'água da embalagem. Quanto menor a taxa de transmissão, menor o deficit de pressão de vapor d'água e maior a umidade relativa no interior da embalagem, o que reduz a taxa de transpiração das frutas. De maneira geral, não se constatou influência dos tratamentos sobre os teores de sólidos solúveis das uvas, que permaneceram praticamente constantes em relação ao inicial (12\%). De forma semelhante, não se observou influência significativa da atmosfera modificada sobre a acidez titulável dos frutos e a relação SST/AT. Somente aos 28 dias de armazenamento a $1^{\circ} \mathrm{C}$, observou-se maior teor de sólidos solúveis totais nos cachos testemunha, provavelmente em razão da maior perda de massa de matéria fresca das bagas, enquanto os cachos embalados em PELBD $50 \mu \mathrm{m}$ apresentaram os menores teores, o que pode ter ocorrido em razão das condições de anaerobiose promovidas por esta embalagem, que podem ter levado os frutos a produzir energia pela respiração anaeróbica (Figura 1). Sob tal aspecto, Artés-Hernández et al. (2006) relataram que o acondicionamento de uvas 'Superior Seedless' em filmes de polipropileno, por 7 dias a $0^{\circ} \mathrm{C}$, seguido por 4 dias a $8^{\circ} \mathrm{C}+2$ dias a $20^{\circ} \mathrm{C}$, não acarretou alterações significativas nos teores de sólidos solúveis totais e acidez titulável, e foi eficiente na redução da perda de massa de matéria fresca e incidência de podridões.
Quanto à avaliação dos efeitos da atmosfera ativa e passiva, na conservação de uvas 'Niagara Rosada' com utilização do filme PEBD $25 \mu \mathrm{m}$, foi observado que, em armazenamento refrigerado, os níveis médios de $\mathrm{O}_{2}$ foram $18,44,18,80,19,15$ e $19,86 \%$, com uso de PEBD $25 \mu \mathrm{m}$, PEBD $25 \mu \mathrm{m}\left(21 \% \mathrm{O}_{2} / 5 \% \mathrm{CO}_{2}\right)$ e PEBD $25 \mu \mathrm{m}\left(21 \% \mathrm{O}_{2} / 10 \% \mathrm{CO}_{2}\right)$, PEBD $25 \mu \mathrm{m}$ $\left(21 \% \mathrm{O}_{2} / 20 \% \mathrm{CO}_{2}\right)$, respectivamente, enquanto os níveis médios de $\mathrm{CO}_{2}$ foram $1,10,1,14,1,10$ e $0,94 \%$ (Figuras 2 A e 2 B). A transferência dos frutos para $25^{\circ} \mathrm{C}$ causou aumento imediato nas concentrações de $\mathrm{CO}_{2}$ de 4,15\% (PEBD $25 \mu \mathrm{m}$ ), 4,11\% (PEBD $25 \mu \mathrm{m}$ $21 \% \mathrm{O}_{2} / 5 \% \mathrm{CO}_{2}$ ), 3,22\% (PEBD $25 \mu \mathrm{m}-21 \% \mathrm{O}_{2} / 10 \%$ $\mathrm{CO}_{2}$ ) e $3,61 \%$ (PEBD $25 \mu \mathrm{m}-21 \% \mathrm{O}_{2} / 20 \% \mathrm{CO}_{2}$ ). Constatou-se também redução nas concentrações de $\mathrm{O}_{2}$ de $8,19,8,21,12,49$ e 9,92\%, com uso de PEBD $25 \mu \mathrm{m}$, PEBD $25 \mu \mathrm{m}\left(21 \% \mathrm{O}_{2} / 5 \% \mathrm{CO}_{2}\right)$, PEBD $25 \mu \mathrm{m}(21 \%$ $\left.\mathrm{O}_{2} / 10 \% \mathrm{CO}_{2}\right)$ e PEBD $25 \mu \mathrm{m}\left(21 \% \mathrm{O}_{2} / 20 \% \mathrm{CO}_{2}\right)$,

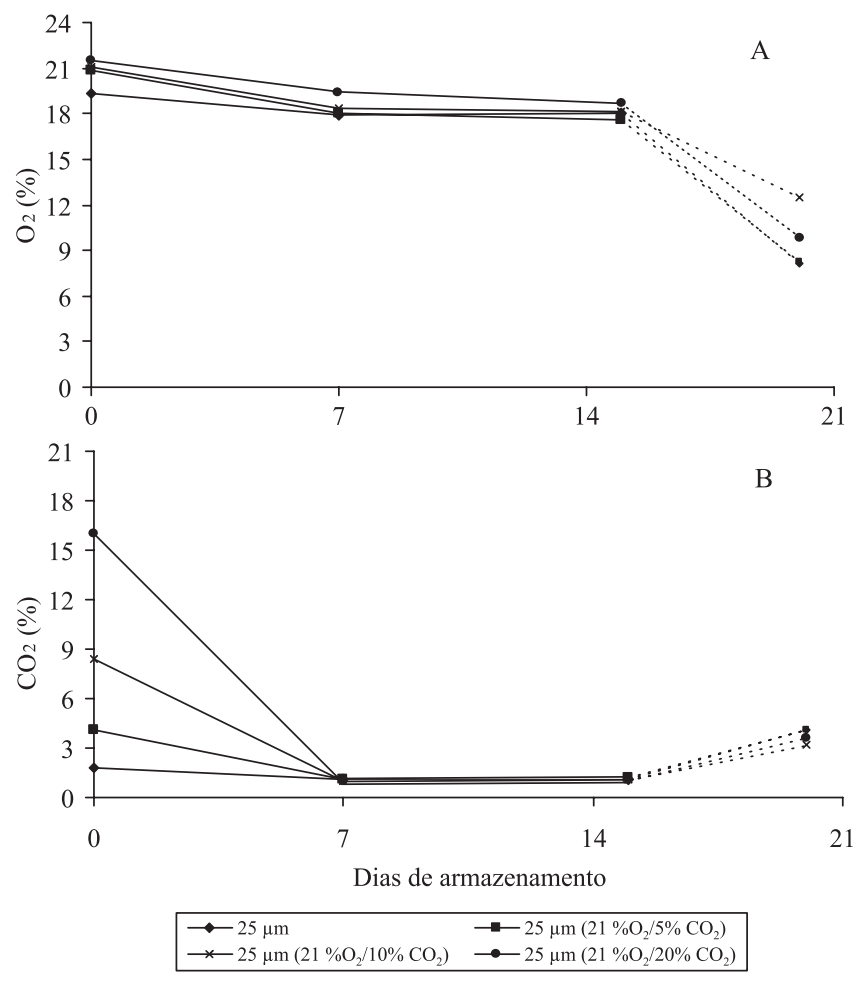

Figura 2. Concentrações de $\mathrm{O}_{2}(\mathrm{~A})$ e $\mathrm{CO}_{2}$ (B) no espaço-livre das embalagens de polietileno de baixa densidade (PEBD), sem injeção de mistura gasosa $(25 \mu \mathrm{m})$, ou com diferentes misturas de gases $\left(21 \% \mathrm{O}_{2} / 5 \% \mathrm{CO}_{2}, 21 \% \mathrm{O}_{2} / 10 \% \mathrm{CO}_{2}\right.$, $21 \% \mathrm{O}_{2} / 20 \% \mathrm{CO}_{2}$ ), durante o armazenamento refrigerado de uvas 'Niagara Rosada' $\left(1^{\circ} \mathrm{C}\right.$ e $90 \%$ de umidade relativa), seguido de um período de cinco dias sob condições do ambiente $\left(25^{\circ} \mathrm{C}\right.$ e $80 \%$ de umidade relativa; linha pontilhad). Experimento II. 
respectivamente, após transferência dos cachos para armazenamento a $25^{\circ} \mathrm{C}$. Portanto, os sistemas de embalagens utilizados não proporcionaram anaerobiose decorrente das baixas concentrações de $\mathrm{O}_{2}$ que, aliadas às altas concentrações de $\mathrm{CO}_{2}$, poderiam prejudicar a qualidade dos frutos pelo acúmulo de etanol e acetaldeído.

O material da embalagem deve apresentar uma taxa de permeabilidade ao $\mathrm{O}_{2}$ que compense o consumo deste gás e uma taxa de permeabilidade ao $\mathrm{CO}_{2}$ que permita a saída deste. Portanto, os teores de $\mathrm{O}_{2}$ e $\mathrm{CO}_{2}$ não devem ultrapassar os limites de tolerância do fruto a esses gases (Zagory \& Kader, 1988). A exposição de um fruto a concentrações de $\mathrm{O}_{2}$ ou $\mathrm{CO}_{2}$, abaixo ou acima, respectivamente, de seu limite de tolerância, resultará em estresse aos tecidos do fruto, manifestado por vários sintomas como amadurecimento irregular, iniciação e agravamento de certos distúrbios fisiológicos, desenvolvimento de sabor e odor desagradáveis e aumento da suscetibilidade a doenças (Kader, 1986).

As diferentes atmosferas, geradas pelos diferentes sistemas de embalagem, não se mostraram efetivas na redução do esbagoamento e da incidência de podridões. Entretanto, o acondicionamento dos cachos em PEBD $25 \mu \mathrm{m}$, sob atmosfera passiva ou ativa, reduziu de forma significativa a perda de massa da matéria fresca dos cachos, após o período de armazenamento refrigerado (Tabela 2). Quando os frutos foram transferidos para armazenamento a $25^{\circ} \mathrm{C}$, observou-se que todos os sistemas de embalagem aumentaram a incidência de podridões nos cachos, o que foi significativamente diferente da testemunha para aqueles acondicionados em PEBD $25 \mu \mathrm{m}\left(21 \% \mathrm{O}_{2} / 5 \%\right.$ $\mathrm{CO}_{2}$ ), provavelmente em razão da elevada umidade no interior das embalagens. De forma semelhante ao constatado para os frutos acondicionados sob refrigeração, observou-se que todos os sistemas de embalagem reduziram de maneira significativa a percentagem de perda de massa de matéria fresca dos cachos após a transferência para $25^{\circ} \mathrm{C}$.

Após o período de armazenamento dos cachos de uva 'Niagara Rosada' sob refrigeração, observou-se que as diferentes atmosferas, obtidas com a utilização de PEBD, aliadas ou não à injeção de mistura gasosa, alteraram alguns dos atributos de qualidade avaliados (Tabela 3). O teor de sólidos solúveis totais foi significativamente menor nos frutos acondicionados sob atmosfera modificada ativa com $20 \% \quad \mathrm{CO}_{2}$

Tabela 2. Esbagoamento, incidência de podridões e perda de massa de matéria fresca em uvas 'Niagara Rosada', acondicionadas em atmosfera modificada passiva e ativa e armazenadas por 15 dias a $1^{\circ} \mathrm{C}$ e $90 \%$ de umidade relativa, seguidos de mais cinco dias em condições do ambiente $\left(25^{\circ} \mathrm{C} \text { e } 80 \% \text { de umidade relativa }\right)^{(1)}$.

\begin{tabular}{|c|c|c|c|c|}
\hline $\begin{array}{l}\text { Tempo de } \\
\text { armazenamento (dias) }\end{array}$ & Tratamento & $\begin{array}{c}\text { Esbagoamento } \\
(\%)\end{array}$ & $\begin{array}{c}\text { Podridões } \\
(\%)\end{array}$ & $\begin{array}{c}\text { Perda de massa de matéria } \\
\text { fresca }(\%)\end{array}$ \\
\hline 0 & - & 0 & 0 & 0 \\
\hline \multirow{5}{*}{15} & Testemunha & $0,07 \mathrm{a}$ & $0,51 \mathrm{a}$ & $1,51 \mathrm{a}$ \\
\hline & PEBD $25 \mu \mathrm{m}$ & $0,88 \mathrm{a}$ & $0,60 \mathrm{a}$ & $0,00 \mathrm{~b}$ \\
\hline & PEBD $25 \mu \mathrm{m}\left(21 \% \mathrm{O}_{2} / 5 \% \mathrm{CO}_{2}\right)$ & $1,02 \mathrm{a}$ & $1,89 \mathrm{a}$ & $0,01 \mathrm{~b}$ \\
\hline & PEBD $25 \mu \mathrm{m}\left(21 \% \mathrm{O}_{2} / 10 \% \mathrm{CO}_{2}\right)$ & $0,52 \mathrm{a}$ & $0,67 \mathrm{a}$ & $0,03 b$ \\
\hline & PEBD $25 \mu \mathrm{m}\left(21 \% \mathrm{O}_{2} / 20 \% \mathrm{CO}_{2}\right)$ & $0,59 \mathrm{a}$ & $0,90 \mathrm{a}$ & $0,16 \mathrm{~b}$ \\
\hline \multirow[t]{6}{*}{ CV (\%) } & & 41,97 & 54,43 & 8,52 \\
\hline & & gens fechadas & & \\
\hline & Testemunha & $2,82 \mathrm{a}$ & $4,02 \mathrm{~b}$ & $5,28 \mathrm{a}$ \\
\hline & PEBD $25 \mu \mathrm{m}$ & $6,19 \mathrm{a}$ & $10,29 \mathrm{ab}$ & $0,57 \mathrm{~b}$ \\
\hline & PEBD $25 \mu \mathrm{m}\left(21 \% \mathrm{O}_{2} / 5 \% \mathrm{CO}_{2}\right)$ & $4,69 \mathrm{a}$ & $17,03 \mathrm{ab}$ & $0,63 b$ \\
\hline & PEBD $25 \mu \mathrm{m}\left(21 \% \mathrm{O}_{2} / 10 \% \mathrm{CO}_{2}\right)$ & $2,04 \mathrm{a}$ & $6,78 \mathrm{ab}$ & $0,63 b$ \\
\hline \multirow[t]{6}{*}{$15+5$} & PEBD $25 \mu \mathrm{m}\left(21 \% \mathrm{O}_{2} / 20 \% \mathrm{CO}_{2}\right)$ & $3,52 \mathrm{a}$ & $7,02 \mathrm{ab}$ & $0,79 \mathrm{~b}$ \\
\hline & & lagens abertas & & \\
\hline & PEBD $25 \mu \mathrm{m}$ & $5,95 \mathrm{a}$ & $9,39 \mathrm{ab}$ & nd \\
\hline & PEBD $25 \mu \mathrm{m}\left(21 \% \mathrm{O}_{2} / 5 \% \mathrm{CO}_{2}\right)$ & $1,08 \mathrm{a}$ & $16,85 \mathrm{a}$ & nd \\
\hline & PEBD $25 \mu \mathrm{m}\left(21 \% \mathrm{O}_{2} / 10 \% \mathrm{CO}_{2}\right)$ & $3,71 \mathrm{a}$ & $8,51 \mathrm{ab}$ & nd \\
\hline & PEBD $25 \mu \mathrm{m}\left(21 \% \mathrm{O}_{2} / 20 \% \mathrm{CO}_{2}\right)$ & $2,59 \mathrm{a}$ & $12,56 \mathrm{ab}$ & nd \\
\hline $\mathrm{CV}(\%)$ & & 39,81 & 32,04 & 11,15 \\
\hline
\end{tabular}

${ }^{(1)}$ Médias seguidas de letras iguais, nas colunas, não diferem entre si pelo teste Tukey, a 5\% de probabilidade. PEBD, polietileno de baixa densidade; nd, não determinado. 
Tabela 3. Sólidos solúveis totais (SST), acidez titulável (AT), cor de casca e firmeza de uvas 'Niagara Rosada', acondicionadas em atmosfera modificada passiva e ativa e armazenadas a $1^{\circ} \mathrm{C}$ e $90 \%$ de umidade relativa por 15 dias, seguidos de mais cinco dias sob condições do ambiente $\left(25^{\circ} \mathrm{C} \text { e } 80 \% \text { de umidade relativa }\right)^{(1)}$.

\begin{tabular}{|c|c|c|c|c|c|}
\hline $\begin{array}{l}\text { Tempo de } \\
\text { armazenamento (dias) }\end{array}$ & Tratamento & $\begin{array}{l}\text { SST } \\
(\%)\end{array}$ & $\begin{array}{c}\mathrm{AT} \\
\text { (\% ác. tartárico) }\end{array}$ & $\begin{array}{c}\text { Cor de casca } \\
\qquad(\Delta \mathrm{E})^{(2)}\end{array}$ & $\begin{array}{l}\text { Firmeza } \\
(\mathrm{N})\end{array}$ \\
\hline 0 & - & 13,48 & 0,69 & & 2,19 \\
\hline \multirow{5}{*}{15} & Testemunha & $13,96 \mathrm{a}$ & $0,69 a$ & $4,22 \mathrm{a}$ & $2,46 b$ \\
\hline & PEBD $25 \mu \mathrm{m}$ & $13,28 \mathrm{ab}$ & $0,61 \mathrm{a}$ & $9,40 \mathrm{a}$ & $5,67 \mathrm{a}$ \\
\hline & PEBD $25 \mu \mathrm{m}\left(21 \% \mathrm{O}_{2} / 5 \% \mathrm{CO}_{2}\right)$ & $13,20 \mathrm{ab}$ & $0,60 \mathrm{a}$ & $8,18 \mathrm{a}$ & $1,90 \mathrm{~b}$ \\
\hline & PEBD $25 \mu \mathrm{m}\left(21 \% \mathrm{O}_{2} / 10 \% \mathrm{CO}_{2}\right)$ & $13,44 \mathrm{ab}$ & $0,62 \mathrm{a}$ & $6,26 \mathrm{a}$ & $1,74 \mathrm{~b}$ \\
\hline & PEBD $25 \mu \mathrm{m}\left(21 \% \mathrm{O}_{2} / 20 \% \mathrm{CO}_{2}\right)$ & $12,96 \mathrm{~b}$ & $0,61 \mathrm{a}$ & $9,16 \mathrm{a}$ & $5,53 \mathrm{a}$ \\
\hline \multirow[t]{6}{*}{$\mathrm{CV}(\%)$} & & 3,57 & 9,64 & 36,99 & 40,23 \\
\hline & \multicolumn{5}{|c|}{ Embalagens fechadas } \\
\hline & Testemunha & $13,84 \mathrm{ab}$ & $0,72 \mathrm{a}$ & $5,40 \mathrm{~cd}$ & $1,69 \mathrm{a}$ \\
\hline & PEBD $25 \mu \mathrm{m}$ & $14,22 \mathrm{a}$ & $0,63 \mathrm{abc}$ & $6,95 \mathrm{~cd}$ & $1,81 \mathrm{a}$ \\
\hline & PEBD $25 \mu \mathrm{m}\left(21 \% \mathrm{O}_{2} / 5 \% \mathrm{CO}_{2}\right)$ & $12,36 \mathrm{~cd}$ & $0,56 \mathrm{c}$ & $4,49 \mathrm{~d}$ & $1,83 \mathrm{a}$ \\
\hline & PEBD $25 \mu \mathrm{m}\left(21 \% \mathrm{O}_{2} / 10 \% \mathrm{CO}_{2}\right)$ & $12,16 \mathrm{~d}$ & $0,54 \mathrm{c}$ & $8,09 \mathrm{c}$ & $1,76 \mathrm{a}$ \\
\hline \multirow[t]{6}{*}{$15+5$} & PEBD $25 \mu \mathrm{m}\left(21 \% \mathrm{O}_{2} / 20 \% \mathrm{CO}_{2}\right)$ & $12,96 \mathrm{bcd}$ & $0,54 \mathrm{c}$ & $6,11 \mathrm{~cd}$ & $2,05 \mathrm{a}$ \\
\hline & \multicolumn{5}{|c|}{ Embalagens abertas } \\
\hline & PEBD $25 \mu \mathrm{m}$ & $13,40 \mathrm{abc}$ & $0,70 \mathrm{ab}$ & $6,94 \mathrm{~cd}$ & $1,65 \mathrm{a}$ \\
\hline & PEBD $25 \mu \mathrm{m}\left(21 \% \mathrm{O}_{2} / 5 \% \mathrm{CO}_{2}\right)$ & $12,40 \mathrm{~cd}$ & $0,59 \mathrm{bc}$ & $12,54 \mathrm{a}$ & $2,04 \mathrm{a}$ \\
\hline & PEBD $25 \mu \mathrm{m}\left(21 \% \mathrm{O}_{2} / 10 \% \mathrm{CO}_{2}\right)$ & $13,28 \mathrm{abcd}$ & $0,64 a b c$ & $11,58 \mathrm{ab}$ & $1,77 \mathrm{a}$ \\
\hline & PEBD $25 \mu \mathrm{m}\left(21 \% \mathrm{O}_{2} / 20 \% \mathrm{CO}_{2}\right)$ & $13,24 \mathrm{abcd}$ & $0,70 \mathrm{ab}$ & $8,77 \mathrm{bc}$ & $1,98 \mathrm{a}$ \\
\hline CV (\%) & & 4,35 & 9,64 & 20,82 & 12,83 \\
\hline
\end{tabular}

(1)Médias seguidas de letras iguais, nas colunas e em cada data de análise, não diferem entre si pelo teste Tukey, a $5 \%$ de probabilidade. ${ }^{(2)}$ Diferença de coloração total entre padrão e amostras $-\Delta \mathrm{E}=\left[(\Delta \mathrm{L})^{2}+(\Delta \mathrm{a})^{2}+(\Delta \mathrm{b})^{2}\right]^{1 / 2}-$ avaliação inicial dos cachos: $\mathrm{L}^{*}=32,46, \mathrm{a}^{*}=5,97$ e $\mathrm{b}^{*}=-1,41$. PEBD, polietileno de baixa densidade.

(PEBD $25 \mu \mathrm{m}-21 \% \mathrm{O}_{2} / 20 \% \mathrm{CO}_{2}$ ), enquanto a firmeza das bagas foi maior nos cachos acondicionados em PEBD $25 \mu \mathrm{m}$ e PEBD $25 \mu \mathrm{m}\left(21 \% \mathrm{O}_{2} / 20 \% \mathrm{CO}_{2}\right)$.

Os valores de acidez não foram influenciados por nenhum dos tratamentos. Após a transferência dos cachos para $25^{\circ} \mathrm{C}$, constataram-se menores teores de sólidos solúveis totais, nos frutos mantidos nas embalagens com $21 \% \mathrm{O}_{2} / 5 \% \mathrm{CO}_{2}$ e $21 \% \mathrm{O}_{2} / 10 \% \mathrm{CO}_{2}$, e nos cachos que foram retirados das embalagens que continham $21 \% \mathrm{O}_{2} / 5 \% \mathrm{CO}_{2}$ no momento em que foram transferidos para $25^{\circ} \mathrm{C}$ (Tabela 3). A acidez titulável foi menor em frutos acondicionados a $21 \% \mathrm{O}_{2} / 5 \% \mathrm{CO}_{2}$, $21 \% \mathrm{O}_{2} / 10 \% \mathrm{CO}_{2}$ e $21 \% \mathrm{O}_{2} / 20 \% \mathrm{CO}_{2}$, bem como naqueles que foram retirados das embalagens que continham $21 \% \quad \mathrm{O}_{2} / 5 \% \quad \mathrm{CO}_{2}$ na transferência para $25^{\circ} \mathrm{C}$. A firmeza das bagas não foi influenciada significativamente pelos diferentes sistemas de embalagem, em comparação à testemunha.

Quanto à cor de casca das bagas de uva armazenadas sob refrigeração, observou-se que nenhum dos sistemas de embalagem alterou a coloração, em comparação aos valores iniciais (Tabela 3). Porém, após a transferência dos cachos para condições do ambiente, verificou-se que os frutos retirados das embalagens que continham $21 \% \mathrm{O}_{2} / 5 \% \mathrm{CO}_{2}, 21 \% \mathrm{O}_{2} / 10 \% \mathrm{CO}_{2}$ e $21 \% \mathrm{O}_{2} / 20 \%$ $\mathrm{CO}_{2}$ diferiram significativamente da testemunha.

\section{Conclusões}

1. A utilização de filme de polietileno linear de baixa densidade (PELBD) $25 \mu \mathrm{m}$, com ou sem injeção de mistura gasosa, e de PVC $17 \mu \mathrm{m}$, aliadas ao armazenamento refrigerado $\left(1^{\circ} \mathrm{C}\right.$ e $90 \%$ de umidade relativa), reduz a perda de massa de matéria fresca dos cachos, mas não é eficiente nas reduções de esbagoamento e de incidência de podridões.

2. O filme de polietileno linear de baixa densidade (PELBD) $50 \mu \mathrm{m}$ acarreta elevação do nível de $\mathrm{CO}_{2} \mathrm{e}$ redução do nível de $\mathrm{O}_{2}$ no interior da embalagem.

\section{Referências}

ARTÉS-HERNÁNDEZ, F.; TOMÁS-BARBERÁN, F.A.; ARTÉS, F. Modified atmosphere packaging preserves quality of $\mathrm{SO}_{2}$-free 'Superior seedless' Table grapes. Postharvest Biology and Technology, v.39, p.146-154, 2006. 
BRACKMANN, A.; MAZARO, S.M.; WACLAWOVSKY, A.J. Armazenamento refrigerado de uvas cvs. Tardia de Caxias e Dona Zilá. Ciência Rural, v.30, p.581-586, 2000.

CAMILI, E.C.; BENATO, E.A.; PASCHOLATI, S.F.; CIA, P. Avaliação de quitosana, aplicada em pós-colheita, na proteção de uva 'Itália' contra Botrytis cinerea. Summa Phytopathologica, v.33, p.215-221, 2007.

CARVALHO, C.R.L.; MANTOVANI, D.M.B.; CARVALHO, P.R.N.; MORAES, R.M. Análises químicas de alimentos. Campinas: Instituto de Tecnologia de Alimentos, 1990. 121p.

CASTRO, J.V. de; PARK, K.J.; HONÓRIO, S.L. Emprego de embalagens para conservação pós-colheita de uvas. Revista Brasileira de Engenharia Agrícola e Ambiental, v.3, p.35-40, 1999.

CHERVIN, C.; WESTERCAMP, P.; MONTEILS, G. Ethanol vapours limit Botrytis development over the postharvest life of Table grapes. Postharvest Biology and Technology, v.36, p.319-322, 2005.

CHITARRA, M.I.F.; CHITARRA, A.B. Pós-colheita de frutas e hortaliças: fisiologia e manuseio. 2.ed. rev. amp. Lavras: UFLA, 2005. 785p.

CIA, P.; BENATO, E.A. Controle alternativo com atmosferas modificada e controlada. In: OLIVEIRA, S.M.A. de; TERAO, D.; DANTAS, S.A.F.; TAVARES, S.C.C. de H. (Ed.). Patologia pós-colheita: frutas, olerícolas e ornamentais tropicais. Brasília: Embrapa Informação Tecnológica, 2006. p.247-264.

CIA, P.; BENATO, E.A.; VALENTINI, S.R. de T.; ANJOS, V.D. de A.; PONZO, F.S.; SANCHES, J.; TERRA, M.M. Radiação ultravioleta no controle pós-colheita de Colletotrichum gloeosporioides em uva 'Niagara Rosada'. Bragantia, v.68, p.1009-1015, 2009.

CRISOSTO, C.H.; GARNER, D.; CRISOSTO, D. Carbon dioxide-enriched atmospheres during cold storage limit losses from Botrytis but accelerate rachis browning of 'Redglobe' Table grapes. Postharvest Biology and Technology, v.26, p.181-189, 2002.

DENG, Y.; WU, Y.; LI, Y. Physiological responses and quality attributes of 'Kyoho' grapes to controlled atmosphere storage. LWT - Food Science and Technology, v.39, p.584-590, 2006.

FERREIRA, D.F. Estatística básica. Lavras: UFLA, 2005. 676p.

INSTITUTO DE ECONOMIA AGRÍCOLA. Produção e número de plantas de videira no Estado de São Paulo. Disponível em: $<$ http://www.iea.sp.gov.br>. Acesso em: 24 maio 2007.

KADER, A.A. Biochemical and physiological basis for effects of controlled and modified atmospheres on fruits and vegetables. Food Technology, v.40, p.99-103, 1986.

KADER, A.A.; ZAGORY, D.; KERBEL, E.L. Modified atmosphere packaging of fruits and vegetables. Critical Reviews in Food Science and Nutrition, v.28, p.1-30, 1989.

MAIA, J.D.G. Manejo da videira Niágara Rosada em regiões tropicais. In: SIMPÓSIO MINEIRO DE VITICULTURA E ENOLOGIA, 1., 2002, Andradas. Viticultura e enologia: atualizando conceitos. Caldas: EPAMIG, 2002. p.49-58.

POMMER, C.V.; TERRA, M.M.; PAIOLI-PIRES, E. Cultivares, melhoramento e fisiologia. In: POMMER, C.V. (Ed.). Uva: tecnologia de produção, pós-colheita, mercado. Porto Alegre: Cinco Continentes, 2003. p.109-294.

TECCHIO, M.A.; TERRA, M.M.; CIA, P.; PAIOLI-PIRES, E.J.; MOURA, M.F.; SANCHES, J.; BENATO, E.A.; HERNANDES, J.L.; VALENTINI, S.R. de T.; SIGRIST, J.M.M. Efeito do ácido naftalenoacético e do cloreto de cálcio na redução das perdas pós-colheita em uva 'Niagara Rosada'. Revista Brasileira de Fruticultura, v.31, p.53-61, 2009.

ZAGORY, D.; KADER, A.A. Modified atmosphere packaging of fresh produce. Food Technology, v.42, p.70-77, 1988.

Recebido em 16 de junho de 2010 e aprovado em 20 de setembro de 2010 